\title{
Téoros
}

Revue de recherche en tourisme

\section{Montréal, capitale gastronomique ?}

\section{Jean-Pierre Lemasson}

Volume 25, numéro 1, printemps 2006

Entre la culture du goût et le goût de la culture

URI : https://id.erudit.org/iderudit/1071030ar

DOI : https://doi.org/10.7202/1071030ar

Aller au sommaire du numéro

Éditeur(s)

Université du Québec à Montréal

ISSN

0712-8657 (imprimé)

1923-2705 (numérique)

Découvrir la revue

Citer cet article

Lemasson, J.-P. (2006). Montréal, capitale gastronomique ? Téoros, 25(1), 24-31. https://doi.org/10.7202/1071030ar d'utilisation que vous pouvez consulter en ligne.

https://apropos.erudit.org/fr/usagers/politique-dutilisation/ 


\section{Montréal, capitale gastronomique?}

\section{Jean-Pierre Lemasson}

Si, dans le monde, on assiste à un intérêt croissant à l'égard de la production agroalimentaire et de l'art culinaire, l'engouement qui saisit actuellement Montréal est proprement fascinant. II y a dans l'air une envie gourmande qui semble contaminer tous ses habitants. Une épidémie d'appétits libérés se répand partout en ville; les estomacs imposent leur nouvelle loi, la hâte de souper nous tenaille, le ventre veut sa part de fête. Avec émerveillement, nous découvrons soudain que nos assiettes sont pleines de multiples fruits et légumes, des meilleures viandes, et que nous buvions du vin millésimé ou du thé délicatement cueilli dans les brouillards célestes, toutes ces nourritures terrestres sont magistralement accommodées, harmonisées, transformées par des cuisiniers de plus en plus imaginatifs. Et nous en redemandons, nous en parlons à nos voisins et à nos amis. La rumeur enfle que nous mangeons bien, remarquablement bien à Montréal. Certains le savent au-delà de nos frontières. Serions-nous en train de muter, passant de l'état d'une ville qui fut toujours gourmande à celle qui se délecte, avec un goût mieux formé, des plats de plus en plus raffinés? Pourrions-nous désormais prétendre allécher ces touristes gourmands dont le déplacement ne se justifie que par une expérience de qualité?

Cet article a pour but de montrer que, des grandes villes du monde, nous sommes parmi les plus choyées en ce qui regarde les plaisirs du boire et du manger. Sous l'effet d'un hédonisme nouveau, la ville entière frisonne à la moindre saveur, frémit à la moindre évocation d'un repas passé en bonne compagnie et tremble d'impatience à goûter un cru nouveau. Mais le plaisir ne saurait être complet sans être partagé, sans qu'il soit l'objet en

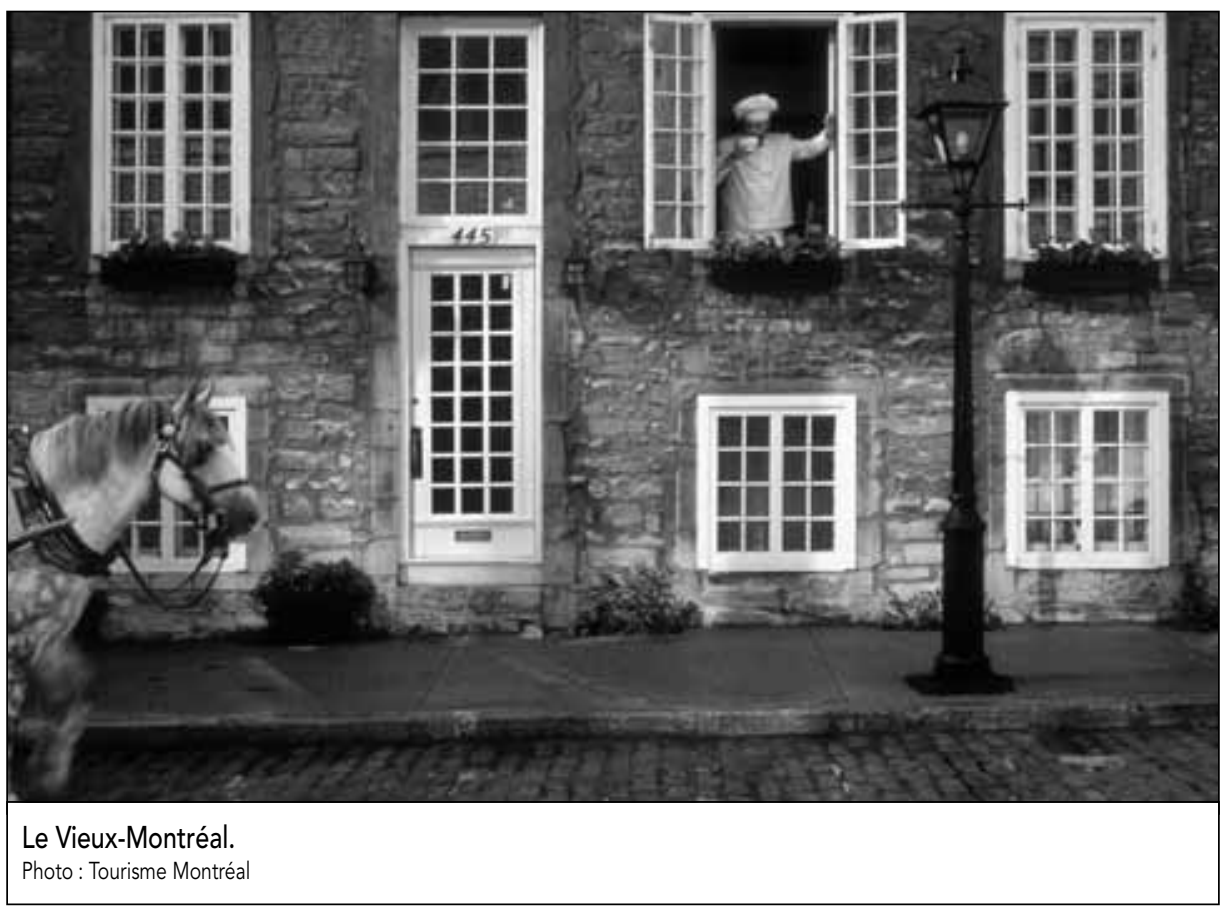

même temps d'un discours qui analyse, enjolive, décerne des éloges, redouble de critique, associant au plaisir de la bouche celui d'une langue bien déliée. Bref, outre de mieux manger, nous tenons à parler du contenu de nos assiettes, enfin capables de considérer la gastronomie comme une affaire sérieuse pour des convives qui ne veulent plus l'être.

Si, comme le dit Pascal Ory, « [...] la gastronomie n'est ni la 'bonne chère' ni la 'haute' cuisine. [Mais] la mise en règle (nomos) du manger et du boire transformée de ce fait 'en arts de la table' »; si " Le gastronome dans son type achevé n'est pas le cuisinier de profession. Il est homme de lettre, au moins en amateur, sa vraie table n'est pas celle où il mange mais son bureau où il écrit [...]" (Ory, 1998: 12), Montréal ne peut-elle prétendre de plein droit à se déclarer ville gastronomique tant le plaisir de manger et de boire est désormais objet de considérations publiques ? Écrire cet article, c'est répondre à la question et, bien entendu, lancer une invitation ouverte à toux ceux qui cherchent une destination pour vivre des jours meilleurs!

\section{Montréal, ville gourmande}

\section{Le bon goût de nos ancêtres lointains... et proches}

II faut reconnaître que Montréal commença par le plus dur, puisque le sieur de Maisonneuve tenta, dès l'origine, d'interdire la consommation d'alcool. En vain. Le peuple, qui savait déjà tout sur l'enfer auquel menait, entre autres, le pêché de gourmandise, se disait qu'un repas bien accompagné de vin ne pouvait pas aggraver ses maux à venir. Cette résistance originelle est symptomatique tout à la fois des interdits récurrents qu'une 
certaine élite catholique, et par la suite protestante, a toujours tenté d'imposer et d'un authentique épicurisme populaire qui ne s'en est jamais laissé conter. II faut dire que la nature généreuse du territoire encourageait l'amour de la bonne chère.

Lors d'un voyage en France dans les années 1660, Pierre Boucher, seigneur de Boucherville, expliquait que toutes les céréales, les pois, les lentilles et les autres fèves " venaient bien " au Québec (Lacoursière et Bizier, 1979: 290-293). II faisait même un inventaire impressionnant de légumes qui poussaient dans les jardins, des carottes, des panais, des navets aux épinards, aux choux, aux asperges en passant par les melons, les melons d'eau, les courges, l'oseille, les cardes... Ainsi, dès l'origine de la Nouvelle-France, les terres québécoises, et particulièrement montréalaises, étaient reconnues pour leur fertilité. Un siècle plus tard, dans son journal, Peter Kalm, savant suédois qui vécut quelques mois au Québec, notait aussi que les poires poussaient bien à Montréal et que des vignes y avaient leur place. Bacchus était venu dans les bagages des colons français, non seulement en graines, mais sous forme de bouteilles dont la consommation représentait $12 \%$ du total de la valeur des importations en 1739 (Lafrance, 1992: 14).

Le régime anglais eut le grand mérite de favoriser l'importation des vins de Bordeaux (sans compter les whiskys, les portos...). Son goût légendaire pour les nourritures simples, mais réconfortantes (du fameux roast beef aux diverses pies en passant par les puddings), laissa une profonde empreinte, tout comme les produits exotiques issus de ses colonies (thé, ketchup, cari...). Lentement mais sûrement une cuisine nouvelle émergeait, cuisine métissée et de plus en plus marquée d'ouverture au monde et d'exotisme. De surcroît, les dégustations d'huîtres n'étaient pas rares et les clubs permettaient d'épancher un goût marqué pour les fastes commensaux tout comme pour l'environnement architectural.

Mais c'est au milieu du XIXe siècle que Montréal, notamment grâce à la création de son port, est devenue un carrefour commercial majeur et une véritable ville. La construction des grands hôtels comme l'hôtel Windsor, la gare-hôtel Viger ou encore l'hôtel Queen's ont fait de Montréal un lieu où la restauration de grande qualité s'est épa- nouie, sans compter l'éclosion de nombreux restaurants, souvent tenus par des cuisiniers immigrants, qui ont pris le haut du pavé. L'ouverture de l'Hôtel de France offre un exemple édifiant d'une pléthore de plats sophistiqués de viande (côtelette d'agneau à la Maintenon, ris de veau à la Périgueux) ; de légumes (asperges, fonds d'artichauts, cardons...), tout comme de desserts (gelée d'orange, meringue italienne...), qui démontraient un savoir-faire remarquable. Comme le disent Marc Lafrance et Yvon Desloges (1990: 30) en conclusion de leur article, "[l]es annonces et les commentaires des gastronomes portent à croire que la cuisine française reconquiert les villes québécoises en cette fin de siècle ».

Du point de vue gastronomique, le Québec était déjà une société distincte. La preuve manifeste en est dans les résultats du référendum tenu en 1898 par Wilfrid Laurier pour prohiber l'alcool au Canada. Le Québec, "comme un seul homme", a voté contre, alors que toutes les autres provinces canadiennes d'alors, comme le montre le tableau 1, ont voté en sa faveur (Prévost et al., 1968 : 61). Nous étions déjà des pionniers de l'exception gastronomique en terre d'Amérique!

Plus près de nous, il est important de retenir que le $X X X^{e}$ siècle fut celui d'un mouvement double et contradictoire. D'un côté, les émules d'Escoffier sont venus faire de Montréal une ville dont la réputation gastronomique attirait la bourgeoisie internationale dans les hôtels et les restaurants réputés. Les Américains furent ainsi parmi les fidèles à venir régulièrement se restaurer et, passés les affres de la seconde guerre mondiale, Montréal, en resserrant notamment ses liens avec la France, connut une flambée de gourmandise fort bien illustrée par l'émergence de multiples clubs gastronomiques et œnophiles et par la tenue de salons culinaires que personne en ville ne voulait rater. Montréal connut alors des joies festives fréquentes et renouvelées. L'amour de la gastronomie était pour un nombre croissant de montréalais un mode de vie.

Mais, de l'autre côté, cet élan ne se reflétait pas toujours dans un quotidien profondément marqué de l'américanité. Les assauts de l'industrialisation alimentaire, la nouvelle religion de la salubrité et ses règlements étouffants, le « vite-fait-vite-mangé », participèrent à donner à la nourriture une vision étroitement

\section{Tableau 1}

Résultats du référendum de 1898 sur la prohibition de l'alcool

\begin{tabular}{l|c|c}
\hline Province & Contre & Pour \\
\hline Ontario & & 16000 \\
Nouvelle-Écosse & & 17676 \\
Nouveau-Brunswick & & 12540 \\
Île du Prince-Édouard & & 6160 \\
Manitoba & & 8000 \\
Territoires du Nord-Ouest & & 2166 \\
Colombie anglaise & & 202 \\
Québec & 43888 & \\
\hline
\end{tabular}

Source : Prévost et al., 1968.

rationaliste. On mangeait pour se repaître des calories nécessaires sans penser à goûter, on avalait comme si de rien n'était et l'on passait à autre chose. Bien entendu ce mal, encore trop répandu sur la planète, est notamment à l'origine de la "révolution du palais » et du retour en force du terroir et des produits artisanaux.

C'est pourquoi, aujourd'hui, les tenants d'une gastronomie traditionnelle et ceux de la gastronomie du terroir sont désormais à l'unisson. La volonté de bien manger au quotidien rejoint le repas d'exception. L'ordinaire doit répondre à des exigences de plus en plus grandes de qualité. Les sens, dont le goût, sont remis à l'honneur. Une récente exposition au Centre canadien d'architecture $(\mathrm{CCA})^{1}$, intitulée Sensations urbaines, en témoigne. Montréal, dont la réputation était déjà bien établie en Amérique du Nord, est prête pour que sa table attire les gourmands de tous les continents.

Un peu comme un touriste, découvrons où la ville se fait "chère» et tressaille. Plus qu'un circuit gourmand, il s'agit ici de saisir dans le paysage visible tous les signes qui révèlent cet immense appétit.

\section{Les marchés publics de Montréal}

Depuis quelques années, les quatre marchés publics permanents sont pris d'une heureuse frénésie. Les légumes et les fruits frais, de plus en plus biologiques, s'y déversent en quantité et en qualité croissantes. De nouvelles espèces d'aubergines, de poivrons, d'ail, de betteraves... y font leur apparition et on découvre avec ravissement des carottes blanches ou des radis d'un noir ache- 
vé. Des espèces oubliées ressuscitent, de la pomme de terre bleue au melon de Montréal, en passant par la plus grande trouvaille qu'un compagnon de Champlain, le sieur Lescarbot fit découvrir à l'Europe: le topinambour!

Que dire des fromages dont la qualité est reconnue désormais par toutes sortes de prix étrangers et dont la variété - plus de 250 - fait en sorte que tous les types de pâtes sont possibles? Les fromages de chèvre et brebis ne sont plus des raretés et font désormais partie des plateaux de fromage de tout hôte montréalais qui se respecte! Les fromages au lait cru ont aussi, enfin, droit de prospérer suite au retrait de règlements sanitaires inappropriés.

Les viandes ne sont pas en reste. Du veau de Charlevoix à l'agneau de Kamouraska, en passant par le canard du lac Brome ou le cerf de Boileau, toutes ces bêtes de choix sont présentes dans les boucheries où la coupe française a détrôné, il y a moins de vingt ans, la coupe américaine (à l'exception du fameux T-bone!). Et nous ne parlons pas des cailles royales, des faisans, des perdrix, des cochonnets ou des viandes plus rares ou exotiques, tel le wapiti ou l'autruche.

En un mot comme en mille, la campagne québécoise étale ses nouvelles richesses et suscite un engouement collectif comme nous n'en avions pas connu depuis fort longtemps. Les produits du terroir et ceux d'autres cuisines du monde se livrent avec générosité, suscitant une envie de découverte et d'expérimentation. Pour la soutenir, des démonstrations culinaires attirent les chalands, tout comme toutes sortes de séances de dégustation qui ne font qu'aggraver ce mal dont on ne veut pas se débarrasser!

Du coup, la fréquentation des marchés explose, les jeunes générations s'y montrent tout comme les nouveaux immigrants des quatre coins du monde ; les marchés se rénovent et, fait tout à fait nouveau, les marchés temporaires saisonniers se multiplient partout au Québec, mais aussi dans les quartiers montréalais. De plus, une quinzaine de kiosques, sortes de petits marchés souvent situés à la sortie des stations de métro, permettent de faire ses approvisionnements frais de manière quotidienne.

\section{Les restaurants}

L'univers de la restauration n'est, bien entendu, pas à l'écart de ce mouvement qui a notamment pour effet de précipiter la faillite de bien des propriétaires figés dans des modes passées. Quelque 3371 restaurants, en 2003, offraient un service complet, c'està-dire un service traditionnel (Bottin statistique, 2004 : 73) aux tables. C'est plus du double des restaurants dits à service limité qui relèvent de la restauration rapide (self-service ou fast food) et qui comptaient 1544 établissements. Au total, ce sont donc quelques 4915 restaurants qui avaient pignon sur rue et dont le nombre continue régulièrement de croître.

Avec un ratio de un restaurant traditionnel pour 450 habitants, Montréal n'est donc pas à plaindre. Elle l'est d'autant moins que le taux de croissance des restaurants traditionnels a été plus rapide que celui des restaurants à service limité (11\% contre $9 \%$ ). Même moins marqué que dans l'ensemble de la province, ce phénomène est des plus encourageants, comme on peut le constater à la lecture du tableau 2.
Tableau 2

Nombre de restaurants par catégorie depuis 1998 au Québec

\begin{tabular}{l|l|l|l|l|l|l}
\hline & $\mathbf{1 9 9 8}$ & $\mathbf{1 9 9 9}$ & $\mathbf{2 0 0 0}$ & $\mathbf{2 0 0 1}$ & $\mathbf{2 0 0 2}$ & $\mathbf{2 0 0 3}$ \\
\hline $\begin{array}{l}\text { Service } \\
\text { complet } \\
\begin{array}{l}\text { Service } \\
\text { restreint }\end{array}\end{array}$ & 5164 & 5147 & 5357 & 5642 & 5676 & 5958 \\
$\begin{array}{l}\text { Total des } \\
\text { restaurants }\end{array}$ & 9879 & 9167 & 4370 & 4139 & 4098 & 4160 \\
\hline \begin{tabular}{l} 
Source: Statistique Canada. \\
\hline
\end{tabular}
\end{tabular}

Tandis que le nombre de restaurants dits rapides a diminué en nombre absolu depuis 1998 , les restaurants où se pratiquent la cuisine et la convivialité ont augmenté de quelque $15 \%$. Voilà une tendance dont il y a lieu de se réjouir et que confirme aussi l'accroissement des ventes.

Que dire de la variété! Déjà héritière de plusieurs traditions culinaires, Montréal, surtout depuis l'exposition tenue en 1968, s'est ouverte à tous les exotismes. Avec un peu plus de $18 \%$ de sa population d'origine immigrée en 2001, Montréal est un vaste car-




refour des nationalités et des cultures. Au rythme des vagues successives d'immigration se sont installées les cuisines chinoise, italienne, portugaise et, plus récemment, vietnamienne, libanaise, algérienne, russe, chilienne..., de telle sorte que la métropole offre aujourd'hui, issues de tous les continents, les traditions alimentaires et culinaires de quelque 80 pays. Certains sont des lieux d'initiation à des cuisines peu connues, que I'on pense par exemple à la cuisine tibétaine, sénégalaise (oui, nous mangeons aussi du poulet yassa!), éthiopienne, polonaise, haïtienne... II manque des semaines aux années pour les essayer toutes. Bien que des données fiables fassent défaut, ce serait au moins $12 \%$ des restaurants qui s'afficheraient comme représentant d'une cuisine étrangère (Turgeon et Pastinelli, 2002 : 254).

Il convient donc de se demander de quel type sont les autres restaurants. Les plus rares sont sans conteste les restaurants québécois dont le nombre se compte sur les doigts d'une main. En effet, si vous voulez goûter aux plats traditionnels, voire folkloriques du pays, vous n'aurez certes pas l'embarras du choix. On préférera vous conseiller des restaurants fort nombreux et de très grande qualité dont on ne sait plus la véritable nationalité tant il est difficile de nommer à quel courant culinaire ils appartiennent. Souvent d'inspiration française, imprégnés d'influences asiatiques et apprêtant des produits locaux, ces restaurants de créativité sont parfois catégorisés comme nord-américains ou encore internationaux. Lieux de nouvelles fusions, de nouvelles inventions, ils nous offrent de superbes découvertes. Ici se réinvente constamment le cosmopolitisme culinaire. L'esprit de découverte est en alerte et la ville nous offre d'innombrables laboratoires du goût, non pas sur le mode un peu futuriste de Ferran Adrià, le grand cuisinier catalan, mais bien de métissages raisonnés, d'harmonies et de contrepoints reflétant des nouvelles manières de vivre, ensemble, le goût des altérités.

Nous avons des restaurants dont la cuisine famiiale sans prétention assure la sécurité et le plaisir ; nous avons des restaurants sympas de quartier au goût de l'amitié (sans fumée... c'est meilleur pour les papilles), des restaurants de " m'as-tu-vu-et-je-regarde-les-autrespasser ", des restaurants de poissons superbement grillés, des restaurants de spécialités lyonnaises, baihanaises, toscanes... et des restaurants où l'on apporte son vin.
Et, bien entendu, nous avons des tables qui se comparent aux meilleures du monde. Certes le guide Michelin qui éprouve en France, par les temps qui courent, quelques difficultés de légitimité, ne nous permet pas des comparaisons toujours délicates; il n'en reste pas moins que les guides culinaires d'ici et ceux des pays étrangers, avec des exigences égales, année après année, classent plusieurs de nos restaurants dans les sommets, toutes relatives ces classifications puissent-elles être. La constance des consensus, mais aussi la satisfaction de toutes les exigences de l'expérience gastronomique convergent, que ce soit en ce qui concerne le cadre, le service et le véritable raffinement culinaire, pour que les touristes puissent rester bouche bée. Les noms de Nuances, Toqué !..., sont prononcés avec une révérence, suivis d'une liste considérable où luttent pied à pied L'Épicier, Au Pied de Cochon, Les Caprices de Nicolas, Cube, Les chèvres, Anise, Christophe, Europea...

Fait nouveau au Québec, à Montréal en particulier, les cuisiniers prennent la plume et, pour la première fois, se transforment en... littérateurs. Même si dans notre amour de la bonne chère nous ne pouvons exclure le prolifique Daniel Vézina du restaurant Laurie Raphaël de Québec ou encore Anne Desjardins du restaurant L'eau à la bouche de Sainte-Agathe, qui ont écrit de fort beaux livres, nous devons souligner le travail exemplaire de Laurent Godbout (L'Épicier, Chez Godbout), de Patrice Demers (Les chèvres), de Jérôme Ferrer (Europea). Avec humour, plusieurs cuisiniers se sont aussi prêtés à parler de leurs recettes dans ce qui est, à notre connaissance, le premier livre de cuisine pensé comme une bande dessinée (Pariseau, 2005).

\section{L'apprentissage du bon}

L'intérêt de Montréal pour l'excellent ordinaire ne semble pas une lubie de passage, un moment éphémère d'une passion aussi vite éteinte qu'avivée. La relève s'active déjà aux fourneaux des grands. De nombreux jeunes professionnels sortent désormais d'excellentes écoles, dont l'Institut d'hôtellerie et du tourisme du Québec est le plus beau fleuron. Depuis sa création en 1968, l'institut a formé des générations de chefs qui sont aujourd'hui aux commandes de nombreux restaurants et le seront plus encore à l'avenir. La professionnalisation accélérée de tous les métiers de bouche assure à Montréal une « sécurité gastronomique", si l'on peut dire, toute nouvelle. Les jeunes sont désormais attirés par les fumets de cuisine, tout comme une partie de plus en plus importante de la population, qui suit des cours dans diverses institutions culinaires publiques et privées. Les chefs ne meurent pas de faim, sont recherchés pour mettre du soleil dans les journées des ménagères, mais, surtout, de plus en plus souvent, des travailleurs professionnels qui se détendent ainsi le soir, plutôt que de regarder la télévision. Tout y passe : de la cuisson des pâtes à l'art du wok et, cosmopolitisme aidant, les amateurs de sushi comme de tapas peuvent se mettre au diapason des nouvelles modes alimentaires. Comment maîtriser les techniques de cuisson à la vapeur ou monter les grandes sauces classiques? Pour se faire des amis, il suffit de vouloir apprendre à cuisiner! Les écoles de cuisine sont légion, que ce soit dans le cadre d'instituts de formation spécialisés ou comme activités complémentaires offertes par des magasins de matériel de cuisine, des magasins spécialisés d'alimentation ou même des grandes chaînes d'alimentation. Les chefs des grands hôtels ne sont pas en reste, surtout hors de Montréal, et certains offrent une journée de cuisine à des yuppies tout excités d'envahir ces lieux presque divinisés où les grands cachent leurs tours de main et leurs trucs, pour être sûrs de ne jamais rien rater.

Et pour le boire, le portrait ne diffère pas. Clubs, cercles, groupes œnologiques se sont multipliés ces dernières années. Les portes des universités se sont ouvertes à ce qui est désormais compris comme d'authentiques expériences culturelles. Si nous ne brillons pas par nos vignobles (à l'exception de ce vin de glace dont la réputation mondiale n'est plus à faire), nous avons sans doute dans nos caves et sur nos tables une des plus grandes variétés des vins du monde qui soit. Nous buvons, bon an mal an, quelque 108 millions de litres de vins - soit, en moyenne, 17,4 litres par personne (SAQ, 2005 : 20 et 25), en provenance de tous les pays qui pressent la treille. Et du vin de table aux crus millésimés, gardant une tradition qui remonte à la NouvelleFrance, nous offrons toujours au moins une bouteille de vin à l'hôte qui a eu la bonne idée de nous inviter, de sorte qu'on ne saurait désormais boire le vin sans se recueillir le nez au bord du verre et que le précieux liquide ait été dûment analysé. Nous avons désormais les sens en alerte du connaisseur. 
Dans de telles conditions, on ne s'étonnera pas que renaissent de leurs cendres, sous une forme plus informelle qu'autrefois, les clubs gastronomiques où des groupes d'amis se font régulièrement la popote, chacun montrant tour à tour aux autres de quoi il est capable! Et on comprendra facilement le succès retentissant de Montréal en Lumière.

\section{Montréal en Lumière}

Depuis sept ans, au creux de l'hiver, suivant le calendrier païen du mardi gras, Montréal en Lumière a modernisé les célébrations gastronomico-bacchiques avec un étonnant mois de février, Montréal célèbre le plaisir de la bouche et de l'olfaction rétronasale. C'est la fête de la gourmandise libérée et l'annonce officielle du retour des grandes forces festives. D'un seul élan, toute la ville se met à table et plonge dans la grande expérience culinaire collective. Toutes les papilles sont en alerte pour goûter, évaluer, commenter les plats que les grands chefs d'une région et d'une grande ville du monde choisies dans différents pays concoctent dans les cuisines de leurs homologues québécois. L'an passé, 44 paires de cuisiniers, étrangers et québécois, ont fait équipe pour que la cuisine de l'Émilie-Romagne surprenne nos palais qui en ont vu d'autres. Par ailleurs, Chicago étant la ville invitée, les amateurs de nouvelle cuisine américaine, teintée du soleil de Californie et d'influence espagnole, n'ont pas été déçus des expériences qu'ils ont faites les yeux fersuccès. D'un seul coup, pendant dix jours au

més. À ce grand happening culinaire s'ajoutaient de surcroit des activités publiques de démonstration culinaire, des conférences, des midis à prix fixes ouverts aux bourses plus modestes et d'autres activités consacrées au plaisir toujours éphémère et toujours recherché des nourritures terrestres. Un tel événement, par sa nature, son ampleur, sa variété, mais aussi sa qualité, est unique au Monde.

Quelques grandes villes, qui, comme Montréal, ont des prétentions de rayonnement international, se livrent aussi, mais sur un mode mineur à nos yeux, aux agapes collectives. Plusieurs ont en effet compris que le rayonnement sur la scène internationale peut passer par l'attrait du ventre. Si les villes se battent pour annoncer les événements sportifs, elles peuvent aussi se battre pour appâter les touristes en quête de sensations nouvelles. Melbourne, Singapour et Chicago sont ainsi parmi les principales villes saisies d'un immense besoin festif. Tout le monde s'y donne rendez-vous pour y créer des rassemblements culinaires aux foules parfois énormes. Chicago, au mois de juillet, s'abandonne ainsi totalement aux odeurs des barbecues et à la fine cuisine... du hot dog! La ville sent bon la graisse qui brûle. Fête d'un certain goût si ce n'est de la gastronomie, mais fête orale quand même! Notons que les cuisines dites ethniques y sont aussi convoquées. Melbourne et Singapour sont plus raffinées et l'on ne saurait, en ces lieux, ne pas inviter de grands chefs pour montrer

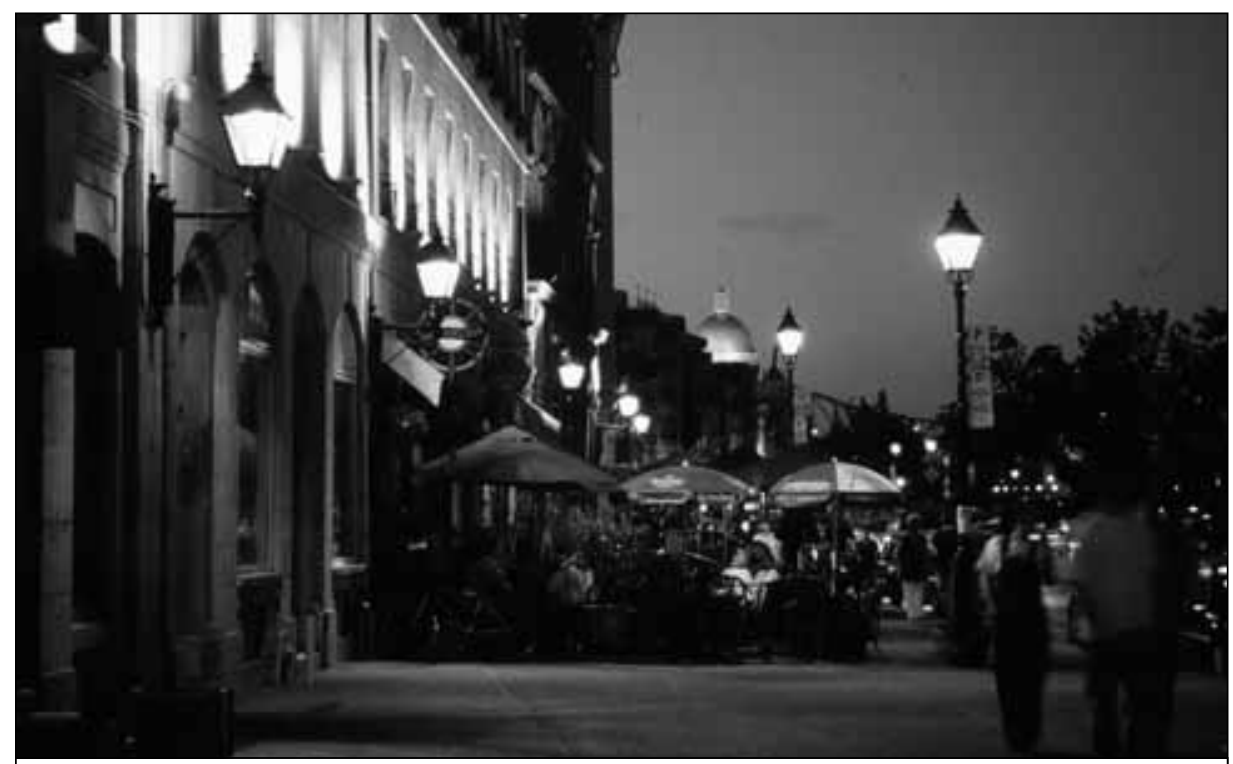

Ambiance d'été dans le Vieux-Montréal.

Photo: Tourisme Montréal aux masses que, entre la bouffe et la cuisine, il y a une différence de savoir-faire et de rituels. Chacun a son motif: Singapour rêve d'inventer une nouvelle cuisine asiatico-européenne et Melbourne fait dans les harmonies gustatives pour mieux exporter son vin. Mais, dans tous les cas, aucune de ces louables initiatives n'a l'envergure - tant sur les plans du cosmopolitisme et de l'ancienneté que de la diversité - trouvée à Montréal. Ce n'est pas du jour au lendemain que nous avons subitement inventé cette fête du goût, ce potlatch de la côte est, car les Fêtes gourmandes, qui se sont déroulées pendant dix ans sur l'île Sainte-Hélène, montraient que le désir d'une faim ludique nous travaillait.

Aujourd'hui, l'événement est exceptionnel, car le goût du boire et du manger y rejoint le goût plus large de l'art et la culture. Montréal en Lumière est un événement où les arts de la table, les arts de la lumière et les arts de la scène, tout comme des arts variés présentés dans les musées, se rencontrent, se renforcent, essaient de jouer sur tous les niveaux du goût comme pratique esthétique commune à tous les arts. La manière de le faire varie chaque année et chaque fois les rencontres sont à réinventer par l'intermédiaire des activités et des artistes différents. Rien n'est vraiment jamais acquis, sinon ce mouvement de recherche d'émotions nouvelles, d'expériences inédites... de transgressions ludiques indispensables à l'esprit des fêtes, comme ces visites des musées ouverts, pour l'occasion, jusqu'aux petites heures du matin.

Le résultat net est le suivant, en termes de fréquentation: des quelque 200000 personnes qui, à l'origine, participaient aux fêtes, toutes activités confondues, nous sommes passés à plus de 500000 en 2004 (Montréal en Lumière, 2004)! Devant une telle participation, force est de reconnaître que même si tout le monde ne salive pas à l'unisson, une bonne partie de la ville rêve de cuisson sous vide et de chefs aux habits d'un blanc parfait. Bien que les statistiques soient plutôt des estimés, ce serait presque 60000 personnes qui, à cette période, transforment nos restaurants en ruches bourdonnantes (Montréal en Lumière, 2004). Toutefois, en cette matière, le critère de réussite est le regard de l'amoureux qui glisse de sa conjointe vers son assiette et y reste béatement fixé pour au moins une demi-seconde d'éternité! Allez-y et vous verrez : les yeux de certains clients hésitent entre deux mondes! 


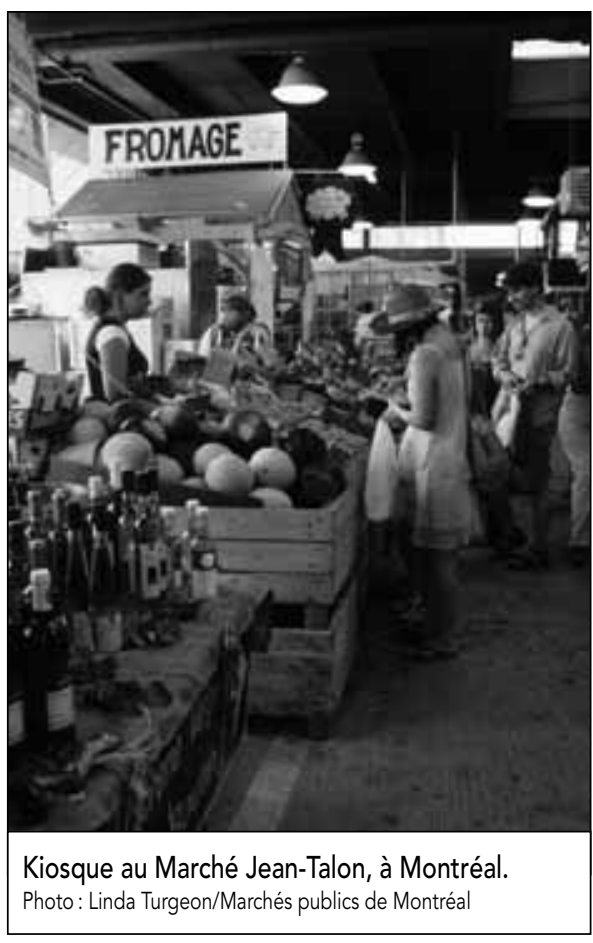

Ce que révèle Montréal en Lumière sur nous-mêmes est non seulement cet amour de la chère qui désormais ne nous quitte plus, mais aussi que notre cosmopolitisme est profond et désormais guidé par une véritable faim d'exotisme culinaire. Nous aimons manger toutes les cuisines du monde et nous aimons plus encore rêver manger toutes les cuisines du monde. Pour nous, l'exotisme est une forme de liberté, un voyage permanent dans notre imaginaire toujours migrant. Montréal a en quelque sorte trouvé sa manière de manger, son style, son ambiance, qui en font désormais une ville non seulement particulière, mais à certains égards exceptionnelle.

L'effet sur la ville est profond et perdure audelà du temps des réjouissances. Nous pouvons en effet, inspirés par les meilleurs exemples, amplifier une dynamique de création culinaire déjà bien vivante. Les clients en redemandent. II y a une forme de complicité vertueuse qui s'installe et affermit de manière décisive le désir de tous de faire de Montréal une grande ville gastronomique. Le cuisinier veut faire mieux d'une année à l'autre et le client veut vivre du nouveau de haute qualité, tous deux désormais de plus en plus liés par une expérience des saveurs renouvelée. Goûter deviendrait-il plus important que manger?

\section{Montréal, capitale gastronomique?}

\section{Une ville qui parle de plus en plus du "gaster ${ }^{2}$ "}

On ne saurait prétendre être une capitale gastronomique sans que «l'objet de nos désirs » soit un sujet qui ait pris toute sa place sur la place publique. II ne s'agit plus seulement d'écriture, mais d'une préoccupation qui mobilise tous les moyens de communication.

Pour la première fois, en 2005, le Salon du livre de Montréal avait pour thème le livre de cuisine. Le thème était alléchant et la fréquentation à la hauteur des espérances. II faut dire que la production québécoise de livres de cuisine ces dernières années est relativement importante, variant de 100 à 120 publications $^{3}$ annuellement, soit presque un nouveau livre tous les deux jours ouvrables! Cette production locale rejoint les flots de livres étrangers sur les rayonnages en constante expansion des libraires. II y en a pour tous les goûts, toutes les tailles, toutes les formes, et les photos plus vraies que nature allèchent la clientèle. Pour rajouter à l'envie, la librairie Oliveri sur le chemin de la Côte-des-Neiges fait aussi restaurant, de telle sorte que les chemins entre théorie et pratique sont parfois très courts! De manière plus large, certaines librairies s'affirment comme de nouveaux lieux de convivialité en prévoyant la possibilité pour les lecteurs de grignoter et de siroter la quintessence d'un café!

Pas un jour ne passe sans que les quotidiens traitent d'un thème directement lié à l'alimentaire ou au culinaire. De multiples chroniques régulières sont consacrées soit à la valorisation des produits du terroir, soit à toutes les déclinaisons possibles de la malbouffe. Parfois jusqu'à couper l'appétit. Les cahiers spéciaux thématiques font les premières pages. Manifestement, le public en veut et en redemande. Si autrefois la critique gastronomique était à peu près le seul endroit où la nourriture était un sujet digne d'intérêt, tout ce qui va de la fourche à la fourchette, comme dit l'expression consacrée, retient désormais l'attention des journalistes. La critique gastronomique des grands journaux elle-même évolue et, de surcroît, se dédouble dans l'édition du vendredi et du samedi, sans compter les chroniques œnologiques qui, elles-mêmes, connaissent le même phénomène.
Au Québec, comme ailleurs, les magazines féminins sont des lieux où les recettes et leurs photos s'étalent depuis des lustres à la devanture des kiosques à journaux. Nous avons toutes les revues gastronomiques imaginables provenant d'Italie, des États-Unis, de France et du Canada anglais. L'édition de magazines spécialisés locaux s'est aussi enrichie ces dernières années de plusieurs titres (Flaveurs, Ricardo...), de telle sorte que, là encore, nous n'avons plus que l'embarras du choix. Celui-ci vire même à la paralysie à certains moments de l'année où des numéros spéciaux couvrent des rayons complets.

Les médias radiophoniques et électroniques ne sont pas en reste. Les émissions consacrées à la cuisine sont désormais présentes toute l'année et, si les émissions changent de style selon la saison, leur nombre ne diminue pas, mais tend aussi à croître. RadioCanada, durant l'automne 2005, offre deux émissions radiophoniques et deux émissions de télévision consacrées à l'alimentation et à la cuisine. Télé-Québec diffuse aussi deux émissions de cuisinedont, une consacrée à la cuisine actuelle et l'autre à I'histoire de la cuisine ou à un thème culinaire. Sans compter là encore toutes les émissions qui, de manière directe ou indirecte, abordent les questions culinaires et des saveurs. Certaines émissions radiophoniques, durant l'été, se font même en direct des marchés le samedi matin.

Si au début du XXe siècle, les guides touristiques étaient peu bavards en matière gastronomique, le rattrapage a été fulgurant et les éditions étrangères et surtout locales permettent aujourd'hui de choisir de manière avertie le lieu de nos prochaines agapes ou de nos achats de produits locaux. Montréal offre désormais à ses citadins, et plus encore aux touristes, ses propres commentaires de la scène et, mieux encore, de - la Cène - locale. Ajoutons en terminant que les sites Web relatifs à la nourriture et à la gastronomie prolifèrent et que Montréal se distingue en cette matière avec un des sites les plus consultés de la Francophonie, "Saveurs du Monde »4.

En somme, les médias de toute nature témoignent de ce Montréal gourmand. Ce faisant, ils avivent l'intensité de la demande, reflétant et créant le même désir par ces effets de miroir dont Narcisse avait le secret. 


\section{Grandes... et bonnes tables}

Les touristes gourmands peuvent donc choisir Montréal en toute connaissance de cause, même si la littérature gastronomique, ici comme ailleurs, vaut surtout par sa force évocatrice. Mais les touristes ne sont pas insensibles à cette ambiance où la nourriture est en passe d'être un culte. Les quelques 1,3 million de touristes américains qui passent par Montréal (Tourisme Montréal, 2004) savent qu'elle est une des villes où l'on mange le mieux en Amérique du Nord et, de surcroît, pour un coût défiant toute concurrence.

Une récente étude réalisée par Tourisme Montréal (2003) montre que les Américains qui viennent au Québec sont, pour $58 \%$ d'entre eux, intéressés à la gastronomie, alors que ce pourcentage dépasse à peine $50 \%$ dans les autres provinces. L'image du Québec, et de Montréal en particulier, est donc fort séduisante en cette matière.

Les Américains ne sont pas les seuls à succomber à la séduction. Les Européens manifestent toujours un étonnement quant à la qualité de notre cuisine et de nos restaurants. Et leur incrédulité s'exprime par : «....ah, je ne savais pas! ».

C'est dire que nous avons des atouts majeurs et qu'il est temps que nous portions la bonne nouvelle à tous les professionnels du tourisme et, plus largement encore, à tous ceux pour qui le bien manger est un fait de civilisation, une expression culturelle tout aussi digne d'attention que les autres formes touristiques! Malheureusement, nous ne sommes pas tous également prêts. Force est de constater que certains milieux ne sont pas convaincus de notre richesse culinaire et de son attractivité. Le meilleur exemple à cet effet est la nouvelle politique du gouvernement en matière de tourisme (Politique touristique du Québec, 2005) qui ne mentionne jamais la gastronomie et les acteurs, comme les restaurants, qui jouent un rôle si essentiel. Pourtant, nous avons à Montréal 6 établissement cotés 4 diamants par le CAA et 1 seul coté 5 diamants. New York a 26 restaurants cotés 4 diamants et 6 cotés 5 diamants. En chiffres absolus, nous pourrions avoir l'impression de déparer. Sachant toutefois que la population de l'agglomération new-yorkaise est de presque 20 millions et celle de Montréal de 3 millions, nous pouvons calculer que, avec une population similaire à celle de New York, nous aurions environ 40 restaurants côtés 4 diamants (comparé à 26) et presque 7 cotés 5 diamants (comparé à 6)! II n'y a donc pas de quoi rougir! Ce même exercice, s'il pouvait être fait avec d'autres grandes villes, nous inclinerait à penser que nous sommes peut-être dans ce que nous pourrions appeler le peloton de tête des villes gastronomiques du monde.

Toutefois, cette manière de se comparer est loin d'être pleinement satisfaisante. D'une part, cette logique ne peut que nous amener à comparer des méga cités où il fait de moins en moins bon vivre (et manger au quotidien) et, d'autre part, à rester dans ce que nous pourrions appeler la logique des critiques gastronomiques qui décernent des étoiles quand c'est beau, bon et très cher. La cuisine d'exception n'existe pas seulement chez ceux qui investissent des fortunes plutôt dans le mobilier que dans la cuisine ou qui, avec un don d'ubiquité étonnant, ont plusieurs restaurants à travers le monde sans plus jamais cuisinier dans aucun. La culture de la distinction sur laquelle reposent les étoiles ou les autres systèmes similaires semble parfois accorder plus d'importance à ce qu'on pourrait appeler les formes extérieures de richesse qu'aux saveurs de l'assiette. Cette conception de la gastronomie a pour effet que de plus en plus de chefs exceptionnels, qui ne veulent pas être dépendants de grands groupes agroalimentaires, s'adressent davantage à une clientèle qui préfère les saveurs au statut social (Renard, 2002).

II nous faut donc comprendre qu'une capitale gastronomique ne s'apprécie pas seulement à travers des systèmes formels d'évaluation qui ont leurs qualités, mais aussi d'évidentes limites. Le culte de la gastronomie, culte qui relève d'une véritable sociologie du culinaire, est à prendre en considération malgré sa complexité et les difficultés actuelles à bien le documenter. Nous sommes néanmoins convaincus qu'à ce titre peu de villes peuvent soutenir la comparaison. II nous revient, en ce sens, de nous mobiliser, tant sur le plan du travail d'analyse à poursuivre que celui d'imaginer des stratégies de valorisation inédites.

\section{Perspective}

Montréal est-elle une capitale gastronomique, un lieu de destination pour le touriste avide, gourmet, gourmand, curieux d'éprouver des sensations nouvelles? Montréal est désormais, à n'en pas douter, une ville qui se met à table dans tous les sens du terme. Pour manger, pour boire et pour parler de l'un et de l'autre entre deux bouchées, entre deux bars à vin, entre la télévision et la radio, entre Internet et les nouveaux CD de cuisine. L'intensité de la passion s'arrime à la longue durée, à un imaginaire qui se transforme durablement. À croire que nous entrons dans une nouvelle religion, celle de la convivialité. En ce sens, nous sommes une ville pleinement gastronomique où désormais les mots et les arts de la table s'appellent les uns les autres dans un plaisir vertueux. Prétendre être une capitale serait peut-être un peu présomptueux, même si certains magazines reconnaissent désormais nos qualités à cet égard ${ }^{5}$. En premier lieu, nous devons encore convaincre chez nous les quelques incrédules qui seraient plus lucides s'ils s'inspiraient de l'aphorisme numéro deux de Brillat-Savarin : "les animaux se repaissent, l'homme mange, l'homme d'esprit seul sait manger ". Mais surtout, en second lieu, force est de reconnaître qu'un immense champ de recherche s'ouvre si l'on souhaite se doter de critères relativement objectifs pour mieux définir et caractériser ce que serait une ville gastronomique. Parmi ceux-là figureront notamment l'importance relative de l'attraction touristique, la qualité de l'expérience gastronomique, sa variété, ses ambiances et bien d'autres critères qui pourraient inspirer, pourquoi pas, la création d'un nouvel index. Mais nous n'en sommes pas là et la prudence reste de mise, même si, à la manière du guide Michelin parlant des restaurants trois étoiles, sans fausse honte et en toute honnêteté, nous pouvons affirmer que Montréal est une ville «qui vaut le déplacement! »

Jean-Pierre Lemasson est professeur au Département d'études urbaines et touristique à l'Université du Québec à Montréal.

\section{Notes}

1 Centre canadien d'architecture de Montréal, Exposition Sensations urbaines, septembre 2005-septembre 2006, Mirko Zardini commissaire. Sous la direction de David Howes, une série de conférences portant sur les cinq sens s'est aussi tenue tous les jeudis du 20 octobre au 8 décembre 2005.

2 Gaster, mot latin désignant l'estomac d'où dérive le mot gastronomie.

3 Les données sont disponibles dans Les statistiques de l'édition au Québec publiées chaque année par la Bibliothèque nationale du Québec. Ces données concernent les ouvrages de cuisine et d'hôtellerie regroupés en une seule catégorie. 
5 Montréal a été classée dans les dix premières villes gastronomiques du monde, en 2001, par la revue Travel and Leisure.

\section{Bibliographie}

Bottin statistique de l'alimentation, Édition 2004, Gouvernement du Québec, 121 p.

Lacoursière, Jacques, et Hélène Bizier (1979), L'alimentation de l'habitant, Nos Racines, $n^{\circ} 15, \mathrm{p}$ 281-300.

Lafrance, Marc, (1992), « De la qualité des vins de France ", Cap-aux-diamants, $n^{\circ} 28$, hiver, p. 14-17.

Lafrance, Marc, et Yvon Desloges (1990), "Les restaurants au $19^{\text {ème }}$ siècle ", Continuité, n 52 , hiver, p. 27-30.
Le tourisme gastronomique, Tourisme Montréal, Direction de la recherche, juillet 2003. Disponible sur le site: [http://www.tourisme-mon treal.org/Download/gastronomie.pdf].

Ministère du tourisme du Québec (2005), Vers un tourisme durable, Politique touristique du Québec, Québec, 37 p.

Montréal en Lumière: Bilan 2004, [http://www. montrealenlumiere.com/Historique/historique -2004/FMLO4-bilan-2004-fr.pdf].

Ory, Pascal (1998), Le discours gastronomique français, Éditions Gallimard/Julliard, Paris, 2002 p.

Pariseau, Charles-Emmanuel Pariseau et al., L'appareil, (2005), Les Éditions de la Pastèque, Montréal, 191 p.

Prévost Robert, Suzanne Gagné, et Michel Phaneuf (1968), Histoire de l'alcool au Québec, Montréal, Éditions Stanké, 239 p.
Renard, Jean-Claude( 2002), La grande casserole, coulisses de la gastronomie, Fayard, Paris, $299 \mathrm{p}$.

Société des alcools du Québec (SAQ), Rapport annuel 2005. [http://www.saq.com/pls/dev saq/generator.pp_afficher_page?P_iden_tran =21974497\&P_modi_url=1205103340\&P_n om_page=info_saq_rapp_annu.saq\&P_tab_ para $=$ VIDE!VIDE\&P_tab_para=P_TEXT_TY PE!3].

Tourisme Montréal, Principales statistiques touristiques sur Montréal 2004, [http://www. tourisme-montreal.org/Download/tmf1205 .pdf].

Turgeon, Laurier, et Madeleine Pastinelli (2002), "Eat the World, Post-colonial Encounters in Québec City's Ethnic Restaurants ", Journal of American Folklore, no 115 (456), 254

\section{TÉQROS Revue de recherche en tourisme}

\section{Appel à textes}

TÉOROS invite tous les chercheurs et tous les professionnels qui œuvrent dans le domaine du tourisme, ou qui s'intéressent au tourisme sous tous ses aspects, à soumettre des articles de nature analytique à la revue. On peut soumettre un article en l'envoyant à :

\section{Bruno Sarrasin}

Directeur et rédacteur en chef TÉOROS

Département d'études urbaines et touristiques Université du Québec à Montréal

C.P. 8888, succ. Centre-Ville

Montréal, Québec, Canada

H3С 3 P8

Courriel : teoros@uqam.ca

Tél. : (514) 987-3000, poste 6959

Publiée trois fois l'an depuis 1982, TÉOROS, revue internationale de recherche en tourisme, se veut un outil intellectuel et professionnel pour les acteurs voués au développement du tourisme. Les problématiques liées aux rapports entre le tourisme et la culture ou entre le tourisme et la société, à la gestion ou à la planification en tourisme, à des cas particuliers de mise en tourisme ainsi qu'aux questions méthodologiques d'actualité, par exemple, comptent parmi les sujets abordés. Téoros souhaite étendre le champ de ces investigations en lançant un appel élargi à ses lecteurs pour constituer, dans chacun de ses numéros, mais indépendamment de la thématique adoptée, un regroupement de tels articles de nature analytique.

Les textes soumis doivent apporter une contribution scientifique originale, que ce soit par le biais d'information factuelle jusqu'alors inconnue ou par une nouvelle interprétation d'un thème particulier. Téoros vise avant tout le transfert des connaissances; son objectif est donc de promouvoir une meilleure compréhension des phénomènes liés au tourisme auprès d'un lectorat élargi.

Les auteurs doivent faire parvenir un manuscrit présenté selon les règles de la revue, que l'on peut consulter à www.teoros.uqam.ca. Habituellement, un article analytique compte environ 4000 mots et excè- de rarement 6000 mots, avec deux illustrations; on pourra cependant considérer des textes plus longs ou plus courts.

Les articles peuvent être soumis en anglais ou en français et doivent être accompagnés d'un résumé de 100 à 200 mots, dans la langue de l'article.

Les auteurs qui n'ont pas accès au site Internet de la revue peuvent contacter la rédaction pour obtenir copie des règles de présentation qu'ils devront suivre, quant aux références, notamment. Le manuscrit doit être fourni sur support informatique en format RTF.

Tous les manuscrits seront évalués par un comité de lecture qui décide des articles qui seront publiés. Le comité peut faire des suggestions ou demander des modifications. La rédaction transmettra l'avis du comité aux auteurs et s'assurera que les modifications demandées seront apportées. La réponse du comité est normalement donnée dans les trois mois suivant la soumission d'un article. 